\title{
String Based New Operations -Find and Replace by New Operational Transformation Algorithms for Wide-Area Collaborative Applications
}

\author{
Santosh Kumawat \\ M.Tech Scholar \\ Poornima College of Engg. \\ Jaipur, Rajasthan, India
}

\author{
Ajay Khunteta \\ Asst Prof. Dept. of CS \\ Poornima College of Engg. \\ Jaipur, Rajasthan, India
}

\begin{abstract}
Operational transformation (OT) is an established optimistic consistency control method in collaborative applications. This approach requires correct transformation functions. In general all OT algorithms only consider two character-based primitive operations and hardly two or three of them support string based two primitive operations, insert and delete. In this paper we propose new algorithms that consider first time in history more new string operations that are Find and replace in addition to primitive operations like insert and delete. In history we are having first time algorithms for composite string operation Find and replace. These algorithms for new Find and replace string operations also support earlier algorithms for primitive string operations-insert and delete. It also handles overlapping and splitting of operations when concurrent operations are transformed. These algorithms can be applied in a wide range of practical collaborative applications.
\end{abstract}

\section{General Terms}

Operational transformation (OT), optimistic consistency control method, Find-replace string operations.

\section{Keywords}

Operational transformation, transformation functions, string operations, Find and replace string operations, real-time cooperative editing systems.

\section{INTRODUCTION}

Operational Transformation (OT) [1] is an established optimistic consistency control method in collaborative applications network. Consistency control in this environment must not only guarantee convergence of replicated data, but also attempt to preserve intentions of operations. Fast local response and timely group awareness are accepted performance metrics in group editors. In general optimistic consistency control on linear data structures is done. In this context a family of optimistic concurrency control algorithms called OT has been well established. OT allows building real time groupware tools by correct transformation functions.

The objective of a collaborative environment [10] is to facilitate team working and, in particular, to enable a group of persons to manipulate shared objects, and modify them in a coherent manner. There are many collaborative activities, examples being simultaneous writing of a document by different authors, or cooperative design. In general, an object involved in a collaborative activity is submitted to concurrent accesses and real-time constraints. The real-time aspect necessitates every user seeing the effects of his own acts on the object immediately, and the effects of the acts of other users almost immediately. Consequently, the problem is to conciliate both real-time and consistency constraints, as the object may be modified concurrently by many users. To satisfy these requirements, it is necessary [16] [17] that concurrency control does not use a blocking protocol which could defer user actions.

Cooperative editing systems [12] are very useful groupware tools in the rapidly expanding areas of CSCW. They can be used to allow physically dispersed people to edit a shared textual document, to draw a shared graph structure, to record ideas during a brainstorming meeting, or to hold a design meeting. The goal of our research is to investigate, design, and implement cooperative editing systems with the following characteristics: (1) real-time: the response to local user actions is quick (ideally as quick as a single-user editor), and the latency for reflecting remote user actions is low (determined by external communication latency only); (2) distributed: cooperating users may reside on different machines connected by different communication networks with nondeterministic latency; (3) unconstrained: multiple users are allowed to concurrently and freely edit any part of the document at any time, in order to facilitate free and natural information flow among multiple users.

A plethora of OT algorithms have been proposed over the past two decades. Most of OT algorithms are developed under the framework of Sun et al [5], which includes an informal condition called "intention preservation". As a consequence, in general their correctness cannot be formally proved. In general all OT algorithms only consider two character-based primitive operations and hardly two or three of them support string based two primitive operations, insert and delete. In real collaborative applications in which string based operations are common. The handling of string operations is very intricate, as confirmed in [5]. So there is an open challenge to handle more string operations.

To address the above challenges, this paper proposes a group of OT algorithm. It is based on the ABT framework [15, 17] which formalizes two correctness condition, causality and admissibility preservation. Causality preservation needed whenever an operation o is executed at a site, all operations that happen before o must have been executed at that site. Conceptually, admissibility requires that the execution of every operation not violate the relative position of effects produced by operations that have been executed so far. In general the ABT framework algorithms can be formally proved. The new 
proposed algorithms as per our knowledge first time in history are handling string operations like Find and replace in addition to primitive operations insert and delete and handles overlapping and splitting of operations when concurrent operations are transformed. These algorithms can be applied in a wide range of practical collaborative applications that require atomic string operations.

\subsection{OT Functions- Inclusion and Exclusion Transformation}

OT functions used in different OT systems may be named differently, but they can be classified into two categories.

One is Inclusion Transformation (or Forward Transformation): $\mathrm{IT}\left(\mathrm{O}_{\mathrm{a}}, \mathrm{O}_{\mathrm{b}}\right)$ or $\mathrm{T}\left(\mathrm{op}_{1}, \mathrm{op}_{2}\right)$, which transforms operation $\mathrm{O}_{\mathrm{a}}$ against another operation $\mathrm{O}_{b}$ in such a way that the impact of $\mathrm{O}_{b}$ is effectively included and the other is Exclusion Transformation (or Backward Transformation) : ET $\left(\mathrm{O}_{\mathrm{a}}, \mathrm{O}_{\mathrm{b}}\right)$ or $\mathrm{T}^{-1}\left(\mathrm{op}_{1}, \mathrm{op}_{2}\right)$, which transforms operation $\mathrm{O}_{a}$ against another operation $\mathrm{O}_{b}$ in such a way that the impact of $\mathrm{O}_{\mathrm{b}}$ is effectively excluded.

\section{Background and Related Work}

To explain string operations and the basic ideas of OT, consider a scenario in which two users, A and B, collaboratively edit a shared document which includes a list of students of a class. The document is replicated at the two sites when the users discuss about it online. Suppose that the list is initially "Ram" and the first position of a string is zero. User A extends the list to "Shyam,Ram" by operation OA = insert( 0, "Shyam,"). At the same time, user B extends the list to "Ram,Raman" by operation OB = insert(3,",Raman"). The two sites diverge before their results are merged. When $A$ receives $\mathrm{OB}$, if the operation were executed as-is, the wrong result "Shy,Ramanam,Ram" would yield in the list of A. The intuition of OT [18] is to transform remote operations to incorporate the effects of concurrent local operations that have been executed earlier. In this scenario, for example, A transforms OB into a form O'B such that O'B can be correctly executed in current state "Shyam,Ram" of site A. Considering the fact that A has inserted a string of six characters on the left side of the intended position of OB, we must shift the position of $\mathrm{OB}$ by six to the right, yielding $\mathrm{O}^{\prime} \mathrm{B}=\operatorname{insert}(9$, "Raman"). Execution of O'B in state "Shyam,Ram" results in the right list of "Shyam,Ram,Raman". On the other hand, when user $B$ receives $\mathrm{OA}$, the operation can be executed as-is in current state of B because the target position of OA is not affected by the execution of OB. This results in list "Shyam,Ram,Raman". Now the two sites converge.

The philosophy of OT is to avoid operation overwriting so as not to lose user interaction results.

\section{System Model and Notations}

A number of collaborating sites are there in a system. The shared data is replicated at all sites when a session starts. Local operations are executed immediately and for local responsiveness, each site submits operations only to its local replica. In the background, local operations are propagated to remote sites. The shared data is like a linear string of atomic characters. Objects are referred to by their positions in the string, starting from zero. It consider two only primitive operations, namely, insert(p, s) and delete(p, s), which insert and delete a string $\mathrm{s}$ at position $\mathrm{p}$ in the shared data, respectively. Any operation o has attributes like o.id is the unique id of the site that originally submits o; o.type is the operation type which is either insert or delete; o.pos is the position in the shared data at which o is applied; o.str is the target string which the operation inserts or deletes. For a operation o, o.pos is always defined relative to some specific state of the shared data.

In the following table1[1] general notations of operation are summarized.

To support string wise transformation, we need to introduce a few more notations. Given any string $s$, notation $|s|$ is the number of characters in $s$. If $0<=\mathrm{i}<\mathrm{j}<=|\mathrm{s}|$, notation $s[\mathrm{i}: \mathrm{j}]$ returns a substring of $s$ starting from position $i$ to position $j-1$. If $j$ is not specified, $s$ [i: ] returns a substring from $i$ to the end. For example, let $s=" a b c "$, then $|s|=3$ and $s[0: 2]=" a b "$ and $s[1:]=" b c "$.

\begin{tabular}{|l|l|}
\hline Notation & Brief Description \\
\hline \hline o.id & the id of site that originally generates $o$ \\
$o . t y p e$ & the operation type of $o$, either ins or del \\
$o . p o s$ & the position of $o$ relative to the data model \\
$o . s t r$ & the string inserted or deleted by $o$ \\
\hline$o_{1} \rightarrow o_{2}$ & $o_{1}$ happens before $o_{2}$ \\
$o_{1} \| o_{2}$ & $o_{1}$ and $o_{2}$ are concurrent \\
$o_{1} \sqcup o_{2}$ & $o_{1}$ and $o_{2}$ are contextual equivalent \\
$o_{1} \mapsto o_{2}$ & $o_{1}$ and $o_{2}$ are contextually serialized \\
\hline$\left[o_{1}, o_{2}\right]$ & an ordered list of two operations \\
$<o_{1}, o_{2}>$ & a 2-operation sequence in which $o_{1} \mapsto o_{2}$ \\
$|L|$ & the number of objects in list/sequence $L$ \\
$L_{1} \cdot L_{2}$ & a concatenated list/seq of two lists/seqs \\
\hline
\end{tabular}

Table 1. A summary of the main notations.

\subsection{Literature Survey}

Research on real-time group editors in the past decade has invented an innovative technique for consistency maintenance, called operational transformation In it presents an integrative review of the evolution of operational transformation techniques, with the goals of identifying the major issues, algorithms, achievements, and remaining challenges. First, it use a linear time interval based logical clock[6] for the same purpose of causality preservation as the more complex vector clock approach in existing operational transformation algorithms. This increases system scalability in terms of accommodating late comers in a dynamic collaboration environment. Second, it solve the dOPT puzzle with a one-dimensional history buffer (as compared to the $\mathrm{N}$-dimensional storage in adOPTed [6]) and the time complexity the TIBOT control algorithm is $\mathrm{O}(\mathrm{n})$ (as compared to $\mathrm{O}\left(\mathrm{n}^{2}\right)$ in GOTO [4][5]). Third, it solve the TP2 puzzle in a fully replicated architecture and without using ET (as compared to GOT [4]) or extra mechanisms (as compared to notification server in [7] and sequencer in [3]). The assumptions it made in it that lead to these results are reasonable in the target application domain of distributed interactive groupware systems. 
The notification mechanism for a group editor consists of two algorithms AnyONE and AnyINE, and a propagation protocol SCOP.

In addition, it contribute a new operational transformation control algorithm SLOT for concurrency control, which is significantly simpler and more efficient than existing algorithms. Furthermore, it is free of state vectors, free of ET transformation functions, and free of the TP2 transformation condition.

It have contributed the theory of operation context and the COT (Context-based OT) algorithm. The theory of operation context is capable of capturing essential relationships and conditions for all types of operation in an OT system; it provides a new foundation for better understanding and resolving OT problems.

To ensure the convergence of the copies while respecting the user intention, it have proposed two new algorithms, called SOCT3 and SOCT4.

A novel state difference based transformation (SDT) approach which ensures convergence in the presence of arbitrary transformation paths.

It proposes an alternative framework, called admissibilitybased transformation (ABT), that is theoretically based on formalized, provable correctness criteria and practically no longer requires transformation functions to work under all conditions. Compared to previous approaches, ABT simplifies the design and proofs of OT algorithms.

Next it is having ABTS for string handling. First, it is based on a recent theoretical framework with formal conditions such that its correctness can be proved. Secondly, it supports two string-based primitive operations and handles overlapping and splitting of operations. As a result, this algorithm can be applied in a wide range of practical collaborative applications.

\section{Algorithms}

In this section we are discussing our new proposed algorithms for replace-Find operations of strings.

$$
\text { Syntax for function replace is }
$$
replace(st1,st2,start,end,occr), where st1 is existing string in document and st 2 is new string by what replacement is to be done, start is starting position from where function start and end is the ending position where function stops. Also occr is the number that is equal to the number of occurances of st 1 for what replacement will be done by function replace. Note st1 and st2 can be of different length. And Find(st1,start,end), it return the position of first occurance of string st1 from start. Here also start is equal to starting position of function and end is ending position of function and st1 is string what get find out by function in given document text . Note start always be equal to or less than end. If start not specified then by default it is starting of text and if end not specified then by default it is end of given text.

\section{Example}

To make clear basic idea of OT, consider a scenario in which two users, A and B, collaboratively edit a shared document which includes the text about facts in world. The document is replicated at the two sites when the user discuss about it online. Suppose the document is initially "SrashtiNirmataRam-Hae", and the first position of string is zero. At site-1 User A modify the document by command replace("Nirmata", "Rachiyata", 0, ,1) , the resulting document is "SrashtiRachiyataRam-Hae". At the same time user B at site-2 extend the document to "SrashtiNirmataRamHanumanHae" by insert(18,"Hanuman"). The two sites diverse before their results are merged.

When $A$ receives $\mathrm{O}_{B}$ if the operation are executed as-is, the wrong result SrashtiRachiyataRaHanumanm- Hae" would yield in the list of A. The intutions of OT[20] is to transform remote operations to incorporate the effects of concurrent local operations that have been executed earlier. In this scenario, for example, A transforms $\mathrm{O}_{\mathrm{B}}$ into a form $\mathrm{O}_{\mathrm{B}}^{\prime}$ such that $\mathrm{O}_{\mathrm{B}}^{\prime}$ can be correctly executed in current state "SrashtiRachiyataRam-Hae" of site A. Considering the fact that A has replaced a string of 7 characters by a string of 9 characters on the left side of the intended position of $\mathrm{O}_{\mathrm{B}}$, we must shift the position of $\mathrm{O}_{\mathrm{B}}$ by 2 to the right, yielding $\mathrm{O}_{\mathrm{B}}^{\prime}=$ insert(20,"Hanuman"). Execution of $\mathrm{O}_{\mathrm{B}}^{\prime}$ in state "SrashtiRachiyataRam-Hae" results in the right list "SrashtiRachiyataRam-HanumanHae". On the other hand, when user $\mathrm{B}$ receives $\mathrm{O}_{\mathrm{A}}$, the operation can be executed as-is in current state of $\mathrm{B}$ because the target position of $\mathrm{O}_{\mathrm{A}}$ is not affected by the execution of $\mathrm{O}_{\mathrm{B}}$. This results in list "SrashtiRachiyataRam-HanumanHae". Now the two sites converge.

The philosophy of O'T' is to avoid operation overwriting so as not to lose user interaction results.

\section{Basic IT Functions}

In the most basic form, function $\mathrm{IT}\left(\mathrm{o}_{1}, \mathrm{O}_{2}\right)$ transforms a primitive operation $o_{1}$ with another primitive operation $o_{2}$ and outputs result $\mathrm{o}_{1}{ }^{\prime}$. The output result can be a composite operation or atomic operation. According to [20], the precondition of $\operatorname{IT}\left(\mathrm{o}_{1}, \mathrm{O}_{2}\right)$ is $\mathrm{o}_{1} \mathrm{U} \mathrm{O}_{2}$ and the postcondition is $\mathrm{O}_{2} \rightarrow \mathrm{o}_{1}^{\prime}$.

Now we are discussing basic IT functions ITIR, ITRI, ITDR, ITRD for our replacement operation replace(st1,st2,start,end,occr). Here $\mid$ st2|-|st1|=pc. Also Find(st1,start,end) returns position of first occurance of st1 from start, let it be ' $p$ '. In ITIR we are passing these pc as parameter. If operation o is replace then o.pos is equal to start parameter of replace function. If IT function return o' then $o^{\prime}$ is new start parameter for replace and Find functions where operation o is replace.

\footnotetext{
Algorithm 1:

$\operatorname{ITIR}\left(o_{1}, o_{2}\right.$, pc, start, end, st1 ): $o_{1}{ }^{\prime}$

1: $\mathrm{o}_{1}{ }^{\prime} \leftarrow \mathrm{o}_{1}$

2: for $(\mathrm{i}=0 ; \mathrm{i}<$ occr and $\operatorname{start}<=$ end $; \mathrm{i}++)$

$3: \mathrm{p} \leftarrow$ Find(st 1 ,start,end)

4: if $(\mathrm{p} !=$ null $)$ then

5: if $\mathrm{pc}>0$ then

6: if $\mathrm{p}<\mathrm{o}_{1}$.pos then

7: $\mathrm{o}_{1}^{\prime} \cdot$ pos $\leftarrow \mathrm{o}_{1}^{\prime} \cdot$ pos $+|\mathrm{pc}|$

8: else if $\mathrm{p}=\mathrm{O}_{1}$.pos and $\mathrm{o}_{2}$. $\mathrm{id}<\mathrm{o}_{1}$. id then
} 


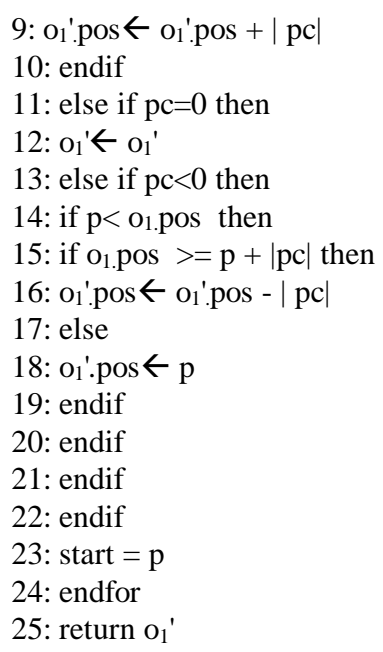

Algorithm 1 transforms operation insertion $\mathrm{o}_{1}$ with another operation that is replacement $\mathrm{O}_{2}$ to incorporate the effects of $\mathrm{O}_{2}$ in $\mathrm{o}_{1}$. Let $\mathrm{s}$ be their common definition state. In it $\mathrm{O}_{2}$ replace a substring st 1 that is already in $\mathrm{s}$ with other new substring st 2 and $\mathrm{o}_{1}$ is to do insertion on $\mathrm{s}$. In it both insertion and replacement are getting operated on same string s.

Here all IT algorithms takes as its parameter $\mathrm{o}_{1}, \mathrm{o}_{2}, \mathrm{pc}$ start, end, st1. In it $\mathrm{o}_{1}, \mathrm{o}_{2}$ are two operations. Also pc is difference in $|s t 1|$ and $|s t 2|$, that is (|st2|-|st1|), where st 1 is string existing in present document and st 2 is new string by what we need to replace st1. Again start is starting position of function and end is ending position of function.

\section{Algorithm 2:}

\section{$\operatorname{ITRI}\left(o_{1}, o_{2}, p c\right.$, start, end, st1 $): o_{1}{ }^{\prime}$}

1: $\mathrm{o}_{1}{ }^{\prime} \leftarrow \mathrm{o}_{1}$

2: $\mathrm{p} \leftarrow$ Find(st1,start,end)

3: if $\mathrm{o}_{2}$.pos $<\mathrm{p}$ then

4: $\mathrm{p}^{\prime} \leftarrow \mathrm{p}+\mid \mathrm{O}_{2}$.str $\mid$

5: else if $\mathrm{p}=\mathrm{O}_{2}$.pos and $\mathrm{o}_{2} . \mathrm{id}<\mathrm{O}_{1}$.id then

6: $\mathrm{p}^{\prime} \leftarrow \mathrm{p}+\mid \mathrm{o}_{2}$ str $\mid$

7: endif

8: return $\mathrm{p}^{\prime}$

Algorithm 2 transforms operation insertion $\mathrm{o}_{2}$ with another operation that is replacement $\mathrm{o}_{1}$ to incorporate the effects of $\mathrm{O}_{2}$ in $\mathrm{o}_{1}$. Let $\mathrm{s}$ be their common definition state. In it $\mathrm{O}_{1}$ replace a substring st 1 that is already in $\mathrm{s}$ with other new substring st 2 and $\mathrm{O}_{2}$ is to do insertion on $\mathrm{s}$. In it both insertion and replacement are getting operated on same string s.

Here $p^{\prime}$ what is getting returned by function ITRI is equal to new start position of replace and Find functions.

\section{Algorithm 3:}

$\operatorname{ITDR}\left(\mathrm{o}_{1}, \mathrm{o}_{2}, \mathrm{pc}\right.$, start, end, st1 ): $\mathbf{o}_{1}{ }^{\prime}$

1: $\mathrm{o}_{1}{ }^{\prime} \leftarrow \mathrm{o}_{1}$

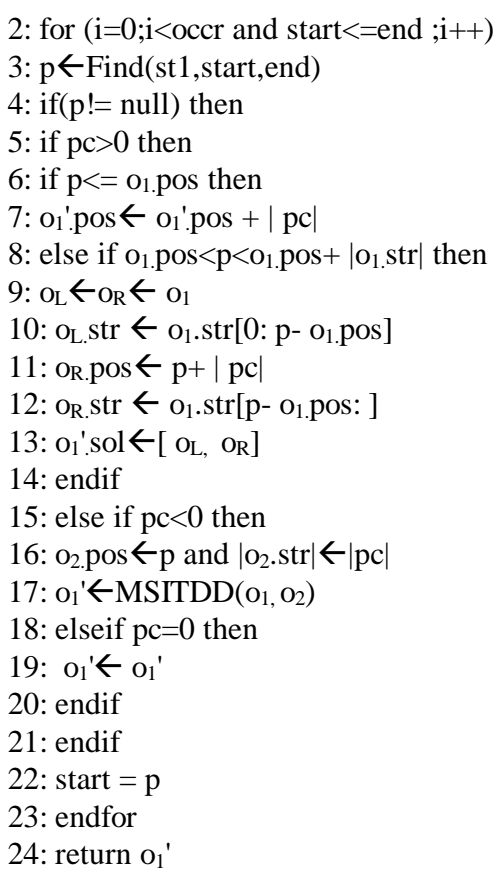

Algorithm 3 transforms operation deletion $\mathrm{o}_{1}$ with another operation that is replacement $\mathrm{O}_{2}$ to incorporate the effects of $\mathrm{O}_{2}$ in $\mathrm{o}_{1}$. Let $\mathrm{s}$ be their common definition state. In it $\mathrm{O}_{2}$ replace a substring st 1 that is already in $\mathrm{s}$ with other new substring st 2 and $\mathrm{o}_{1}$ is to do deletion on $\mathrm{s}$. In it both deletion and replacement are getting operated on same string s.

In Algorithm 3 and Algorithm 4 MSITDD is from our paper[21].

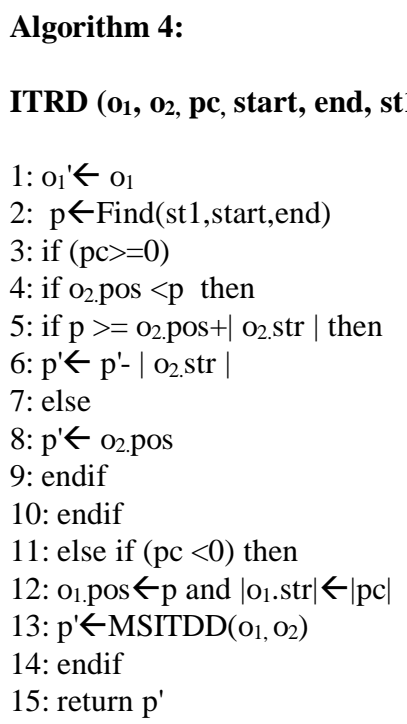

Algorithm 4 transforms operation deletion $\mathrm{O}_{2}$ with another operation that is replacement $o_{1}$ to incorporate the effects of $\mathrm{O}_{2}$ in $\mathrm{O}_{1}$. Let $\mathrm{s}$ be their common definition state. In it $\mathrm{O}_{1}$ replace a substring $\mathrm{st} 1$ that is already in $\mathrm{s}$ with other new substring st 2 and $\mathrm{O}_{2}$ is to do deletion on $\mathrm{s}$. In it both deletion and replacement are getting operated on same string s. 
Here $\mathrm{p}^{\prime}$ what is getting returned by ITRD is equal to new start position of replace and Find functions.

\subsection{Basic Swap Functions}

The basic swapping function for swapping two operations. Given two operations $\mathrm{O}_{1}$ and $\mathrm{O}_{2}$, where $\mathrm{O}_{1} \rightarrow \mathrm{O}_{2}$, function $\operatorname{swap}\left(\mathrm{o}_{1}, \mathrm{o}_{2}\right)$ transposes them into $\mathrm{o}_{1}{ }^{\prime}$ and $\mathrm{o}_{2}{ }^{\prime}$ such that $\mathrm{o}_{2}{ }^{\prime} \rightarrow$ $\mathrm{o}_{1}^{\prime}$. The precondition of $\operatorname{swap}\left(\mathrm{o}_{1}, \mathrm{O}_{2}\right)$ is $\mathrm{O}_{1} \rightarrow \mathrm{O}_{2}$.

Algorithm swapRI and swapRD is to swap replace operation on string with other primitive operations like insertion and deletion on strings.

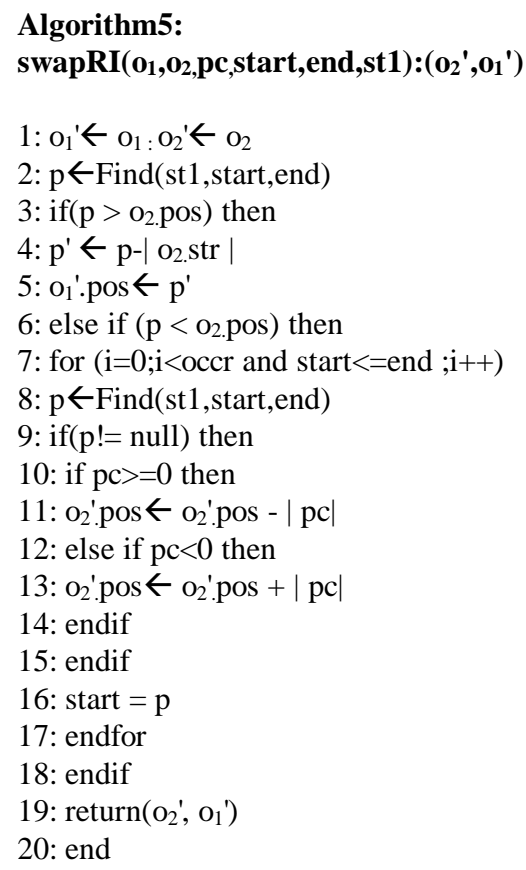

Algorithm 5 swaps replace $\mathrm{o}_{1}$ with an insertion $\mathrm{O}_{2}$. Here it takes as its parameter $\mathrm{o}_{1}, \mathrm{o}_{2}$, pc, start, end, st1 . Also pc is difference in $|s t 1|$ and $|s t 2|$, where st 1 is string existing in present document and st 2 is new string by what we need to replace $s t 1$,so $\mathrm{pc}=(|\mathrm{st} 2|-|\mathrm{st} 1|)$. Again start is starting position of function and end is ending position of function. It returns $\mathrm{o}_{2}{ }^{\prime}, \mathrm{o}_{1}{ }^{\prime}$ that are modified operations where $\mathrm{o}_{2}$.pos is new position for insertion and $o_{1}{ }^{\prime}$.pos is new starting position for modified replace and Find functions.

In Algorithm 6 swaps replace $\mathrm{o}_{2}$ with a deletion $\mathrm{o}_{1}$. Here parameters are like algorithm5. It returns $\mathrm{o}_{2}^{\prime}, \mathrm{o}_{1}{ }^{\prime}$ that are modified operations where $0_{1}$ '.pos is new position for deletion and $\mathrm{o}_{2}$ '.pos is new starting position for modified replace and Find functions. In these swapping algorithms overlapping of replace with insertion or deletion is not considered for reducing extra overhead.

\section{Algorithm 6:}

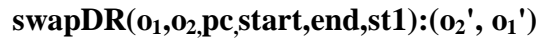

1: $\mathrm{O}_{1}{ }^{\prime} \leftarrow \mathrm{o}_{1}: \mathrm{O}_{2}{ }^{\prime} \leftarrow \mathrm{O}_{2}$

2: $\mathrm{p} \leftarrow$ Find(st 1 ,start,end)

3: if $\left(\mathrm{p}>\mathrm{o}_{1}\right.$.pos $)$ then

4: $\mathrm{p}^{\prime} \leftarrow \mathrm{p}+\left|\mathrm{o}_{1 .} \mathrm{str}\right|$

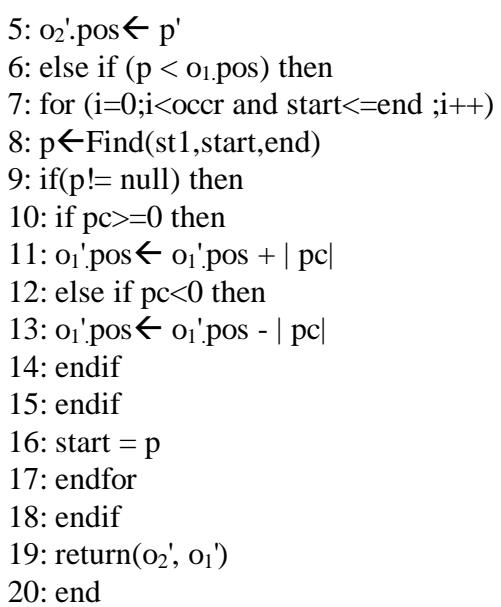

\section{CONCLUSION}

This paper contributes a group of new optimized generic operational transformation algorithms that first time in history consider new composite string operations, Find and replace. It also support existing primitive operations like insert and delete.

First time in history birth of composite string operations like Find and replace in multi user shared environment take place in this paper.

Most of OT algorithms are developed under the framework of Sun et al ${ }^{[5]}$, which includes an informal condition called "intention preservation". As a consequence, in general their correctness cannot be formally proved. In general all OT algorithms only consider two character-based primitive operations and hardly two or three of them support string based two primitive operations, insert and delete.

To address the above challenges, this paper proposes a novel OT algorithm. It is based on the ABT framework [15, 17] which formalizes two correctness condition, causality and admissibility preservation. In general the ABT framework algorithms can be formally proved. The new proposed algorithms first time in history are handling string operations like Find and replace in addition to primitive operations insert and delete and handles overlapping and splitting of operations when concurrent operations are transformed in particular situations. These algorithms can be applied in a wide range of practical collaborative applications that require string operations.

This paper proposed new algorithm like swapRI, swapDR, ITRI ,ITIR, ITDR and ITRD for replace operation of strings, where swapRI and swapDR are basic swap functions and ITRI, ITIR, ITRD and ITDR are basic IT functions for string replace operations.

\subsection{Future Work}

There is a lot of efforts needed to preserve intention preservation and also to preserve semantic consistency and syntactic consistency. There is still scope to extend the support to other composite operations of string handling and char handling. Also it can support other better data structures also. A lot of work is done to reduce space complexity and time 
complexity. Still there is a scope to reduce space complexity and time complexity.

\section{REFERENCES}

[1] ABTS: A Transformation-Based Consistency Control Algorithm for Wide-Area Collaborative Applications Bin Shao, Du Li , Ning Gu . IEEE Paper published in 2009

[2] C. A. Ellis and S. J. Gibbs.Concurrency control in groupware systems. In Proceedings of the ACM SIGMOD'89 Conference on Management ofData, pages 399-407, Portland Oregon, 1989.

[3] H. Yu and A. Vahdat. Design and evaluation of a conit-based continuous consistency model for replicated services. ACM Transactions on Computer Systems, 20(3):239-282, Aug. 2002.

[4] N.Vidot, M.Cart, J.Ferrie, and M.Suleiman. Copies convergence in a distributed realtime collaborative environment. In ACM CSCW'00, pages 171-180, Dec. 2000.

[5] C. Sun, X. Jia, Y. Zhang, Y. Yang, and D. Chen. Achieving convergence, causality- preservation, and intention-preservation in real-time cooperative editing systems. ACM Transactions on Computer-Human Interaction, 5(1):63-108, Mar. 1998.

[6] H. Shen and C. Sun. Flexible notification for collaborative systems. In ACM CSCW'02, pages 77-86, Nov. 2002.

[7] C. Sun and C. Ellis. Operational transformation in real-time group editors: issues, algorithms, and achievements. In ACM CSCW'98, pages 59-68, Dec. 1998.

[8] R. Li and D. Li. A new operational transformation framework for real-time group editors. IEEE Transactions on Parallel and Distributed Systems, 18(3):307-319, Mar. 2007.

[9] G. Oster, P. Urso, P. Molli, and A. Imine. Proving correctness of transformation functions in collaborative editing systems. Technical Report 5795, INRIA, Dec. 2005.

[10] M. Suleiman, M. Cart, and J. Ferrie. Concurrent operations in a distributed and mobile collaborative environment. In IEEE ICDE '98 International Conference on Data Engineering, pages 36-45, Feb. 1998.

[11] C. Sun and C. Ellis. Operational transformation in real-time group editors: issues, algorithms, and achievements. In Proceedings of the ACM Conference on Computer- Supported Cooperative Work, pages 59-68, Dec. 1998.
[12] C. Sun, X. Jia, Y. Zhang, Y. Yang, and D. Chen. Achieving convergence, causality- preservation, and intention preservation in real-time cooperative editing systems. ACM Transactions on Computer-Human Interaction, 5(1):63108, Mar. 1998.

[13] D. Sun and C. Sun. Context-based operational transformation in distributed collaborative editing systems. IEEE Transactions on Parallel and Distributed Systems, 20(10):1454$1470,2009$.

[14] Decouchant D., Quint V., Vatton I.; "L'édition Coopérative de documents avec riffon," Colloque IHM'92, Paris, Décembre 1992.

[15] R. Li and D. Li. Commutativity-based concurrency control in groupware. In Proceedings of the First IEEE Conference

on Collaborative Computing: Networking, Applications and Worksharing (CollaborateCom '05), San Jose, CA,

Dec. 2005.

[16] Ellis C.A., Gibbs S.J., Rein G.L.; "Groupware: Some issues and experiences," Commun. ACM,vol.34, n. 1, pp. 39-59, January 1991.

[17] Greenberg S., Marwood D.; "Real Time Groupware as a Distributed System: Concurrency Control and its Effect on the Interface," in Proc. ACM Int. Conf. on Computer Supported Cooperative Work, Canada, October 1994, pp. 207-217.

[18] C. A. Ellis and S. J. Gibbs. Concurrency control in groupware systems. In Proceedings of the ACM SIGMOD'89 Conference on Management ofData, pages 399-407, Portland Oregon, 1989.

[19] D. Li and R. Li. An admissibility-based operational transformation framework for collaborative editing systems. Computer Supported Cooperative Work: The Journal of Collaborative Computing, Aug. 2009. Accepted.

[20] Prakash A., Shim H. S.; "DistView: Support for Building Efficient Collaborative Applications using Replicated Object," in Proc. ACM Int. Conf. on Computer Supported Cooperative Work, October 1994, pp. 153-164.

[21] Santosh Kumawat and Ajay Khunteta

A Transformation based New Algorithm for Transforming Deletions in String Wise Operations for Wide-Area Collaborative Applications ,International Journal of Computer Applications (0975 - 8887) Volume 4- No.12, Aug 